\title{
Doença renal crônica: doença subdiagnosticada? Análise epidemiológica em um centro de diálise
}

\author{
Chronic kidney disease: underdiagnosed disease? Epidemiological analysis in a dialysis center \\ Enfermedad renal crónica: ¿enfermedad subdiagnosticada? Análisis epidemiológico en un centro
} de diálisis

\author{
Lígia Sant'Ana Dumont \\ ORCID: https://orcid.org/0000-0002-4623-3470 \\ Universidade Evangélica de Goiás, Brasil \\ E-mail: ligiasdumont@gmail.com \\ Isadora Cunha Manata \\ ORCID: https://orcid.org/0000-0001-5188-4879 \\ Universidade Evangélica de Goiás, Brasil \\ E-mail: isadoramanata@hotmail.com \\ Verônica Alcântara Cardoso Duarte Oliveira \\ ORCID: https://orcid.org/0000-0001-5784-0463 \\ Universidade Evangélica de Goiás, Brasil \\ E-mail: veronicaacdo@hotmail.com \\ Maria Luisa Borges Acioli \\ ORCID: https://orcid.org/0000-0003-2063-7810 \\ Universidade Evangélica de Goiás, Brasil \\ E-mail: maluacioli@hotmail.com \\ Guilherme Cristovam Pina \\ ORCID: https://orcid.org/0000-0003-0299-7679 \\ Universidade Evangélica de Goiás, Brasil \\ E-mail: guilhermecpina54@gmail.com \\ Leticia Loureiro Castro Real \\ ORCID: https://orcid.org/0000-0002-6156-7276 \\ Universidade Evangélica de Goiás, Brasil \\ E-mail: leticialcreal@gmail.com \\ Rhaisa Ghannam Macedo \\ ORCID: https://orcid.org/0000-0003-2538-5624 \\ Universidade Católica de Brasília, Brasil \\ E-mail: rhaisamacedo_@hotmail.com
}

\begin{abstract}
Resumo
A doença renal crônica (DRC), caracterizada pela diminuição progressiva da função renal, tem importância e prevalência elevada, mas muitos pacientes são diagnosticados tardiamente, quando a terapia renal substitutiva (TRS) é mandatória, tornando o rastreio da doença valioso para avaliação clínica. Este estudo objetiva analisar a quantidade de pacientes com diagnóstico de DRC de etiologia indeterminada, bem como o tempo decorrido entre a data de diagnóstico da DRC e o início da TRS, salientando a importância do rastreio de DRC em pacientes com fatores de risco, conhecidos ou não. Para isso, foi realizado um estudo observacional, descritivo e retrospectivo de 177 prontuários da Milli Clínica Nefrológica em Aparecida de Goiânia - GO, sendo a população de estudo os pacientes admitidos à diálise no período de 2018 a 2021. As características avaliadas foram: procedência, faixa etária, sexo, fatores de risco para DRC, história familiar de DRC, tempo de tratamento conservador da DRC, data de diagnóstico da DRC, etiologia da DRC e data de admissão à TRS. Evidenciou-se a maioria dos pacientes de procedência de Aparecida de Goiânia (n=116), do sexo masculino $(n=105)$, com faixa etária entre 50-59 anos $(n=49)$, com hipertensão arterial sistêmica $(n=118)$ e/ou diabetes $(n=69)$ e sem tratamento conservador prévio $(n=112)$. Concluiu-se, assim, que a DRC atualmente é uma doença subdiagnosticada e seu diagnóstico precoce é importante para retardar a progressão da doença e a admissão do paciente à TRS.
\end{abstract}

Palavras-chave: Doença renal crônica; Terapia de substituição renal; Diagnóstico precoce.

\section{Abstract}

The chronic kidney disease (CKD), characterized by progressive decrease in renal function, has high importance and prevalence, but many patients are diagnosed late, when renal replacement therapy (RRT) is mandatory, making screening of the disease valuable for clinical evaluation. This study aims to analyze the number of patients diagnosed with CKD of undetermined etiology, as well as the time elapsed between the date of diagnosis of CKD and the onset of 
RRT, emphasizing the importance of CKD screening in patients with risk factors, known or not. Therefore, an observational, descriptive and retrospective study of 177 medical records of the Milli Clínica Nephrological in Aparecida de Goiânia - GO, was carried out, and the study population was the patients admitted to dialysis from 2018 to 2021. The characteristics evaluated were origin, age group, gender, risk factors for CKD, family history of CKD, time of conservative treatment of CKD, date of diagnosis of CKD, etiology of CKD and date of admission to RRT. Most patients are from Aparecida de Goiânia ( $n=116)$, male $(n=105)$, aged between 50-59 years $(n=49)$, with hypertension $(n=118)$ and/or diabetes $(n=69)$ and without previous conservative treatment $(n=112)$. Thus, it was concluded that CKD is currently an underdiagnosed disease, and its early diagnosis is important to delay the progression of the disease and the patient's admission to RRT.

Keywords: Chronic kidney disease; Renal replacement therapy; Early diagnosis.

\section{Resumen}

La enfermedad renal crónica (ERC), caracterizada por una disminución progresiva de la función renal, tiene una gran importancia y prevalencia, pero muchos pacientes se diagnostican tardíamente, cuando la terapia de reemplazo renal (TRR) es obligatoria, lo que hace que el cribado de la enfermedad sea valioso para la evaluación clínica. Este estudio tiene como objetivo analizar el número de pacientes diagnosticados de ERC de etiología indeterminada, así como el tiempo transcurrido entre la fecha del diagnóstico de ERC y el inicio del TRR, destacando la importancia del cribado de ERC en pacientes con factores de riesgo, conocidos o no. Por ello, se realizó un estudio observacional, descriptivo y retrospectivo de 177 historias clínicas de la Milli Clínica Nephrological en Aparecida de Goiânia - GO, y la población de estudio fueron los pacientes ingresados en diálisis de 2018 a 2021. Las caracteristicas se evaluaron origen, grupo de edad, sexo, factores de riesgo de ERC, antecedentes familiares de ERC, tiempo de tratamiento conservador de ERC, fecha de diagnóstico de ERC, etiología de ERC y fecha de ingreso en TRR. La mayoría de los pacientes son de Aparecida de Goiânia $(n=116)$, varones $(n=105)$, de 50 a 59 años $(n=49)$, con hipertensión $(n=118)$ y / o diabetes $(n=69)$ y sin tratamiento conservador previo $(n=112)$. Así, se concluyó que la ERC es actualmente una enfermedad infradiagnosticada, y su diagnóstico precoz es importante para retrasar la progresión de la enfermedad y el ingreso del paciente a TRR.

Palabras clave: Enfermedad renal crónica; Terapia de reemplazo renal; Diagnostico temprano.

\section{Introdução}

A doença renal crônica (DRC) é caracterizada pela diminuição lenta e progressiva da função renal sangue (Vieira et al., 2010). Em sua fase inicial, é, majoritariamente, assintomática, um dos fatores que tornam o diagnóstico precoce um desafio para os médicos (Bastos \& Kirsztajn, 2011). Segundo a última atualização do Kidney Disease: Improving Global Outcomes (KDIGO, 2012), a DRC é definida pela anormalidade da estrutura e/ou função dos rins persistente por mais de três meses com implicação para a saúde, isto é, que cursam com efeitos indesejáveis na qualidade de vida do paciente.

Trata-se de uma doença que vem demonstrando elevada prevalência em estudos recentes. Em 2017, o número de indivíduos com DRC, em seus diferentes estágios, ultrapassou o número de casos de diabetes, osteoartrite e doença pulmonar obstrutiva crônica (DPOC). Entretanto, muitos países ainda não conseguem oferecer o atendimento necessário aos pacientes renais crônicos (Bikbov et al., 2020).

Diante do caráter progressivo da doença, percebe-se a importância de um diagnóstico precoce a fim de evitar um prognóstico desfavorável muitas vezes relacionado ao início precoce de terapia renal substitutiva (TRS) e/ou ausência de preparo prévio para realização dele. Contudo, pelo fato do estágio inicial da DRC cursar de modo assintomático, diagnosticar precocemente essa doença é um desafio para a equipe médica (Bastos \& Kirsztajn, 2011).

Além disso, os parâmetros renais convencionais podem não ser sensíveis o bastante para detectar a doença nos estágios iniciais. Marcadores de função renal, como creatinina e taxa de filtração glomerular (TFG), começam a mostrar alterações em seus níveis tempo depois da patologia já estar estabelecida (Selvarajah et al., 2016). No Brasil, por exemplo, estima-se que 2,9 milhões de brasileiros tenham a TFG reduzida a um terço, comparada a de indivíduos normais (Bastos \& Kirsztajn, 2011).

Diante disso, a importância do rastreio dos fatores de risco da DRC, como hipertensão arterial sistêmica (HAS), diabetes e obesidade, reside no fato de detectar quais são os indivíduos com maior chance de evolução para DRC e que necessitam de uma atenção maior nos exames de triagem (Oliveira et al., 2018). Na obesidade, por exemplo, é encontrado um estado de hiperfiltração e aumento da pressão glomerular na tentativa de atender às demandas do peso corporal, de modo que se comporta 
como um fator que pode tanto desencadear a DRC quanto atuar na sua progressão (Amaral et al., 2019).

É importante ressaltar que os fatores socioeconômicos também precisam ser considerados. O baixo nível socioeconômico está associado à microalbuminúria, macroalbuminúria, redução da TFG e à perda progressiva de função dos néfrons (Marinho et al., 2017).

Tratando-se de DRC, um diagnóstico tardio pode custar o bem-estar físico, emocional e mental do paciente. A hemodiálise, método mais difundido de TRS, traz consigo uma grande piora da qualidade de vida do paciente. Terapia medicamentosa contínua, restrições dietéticas, limitações físicas e nutricionais, afastamento do trabalho e dependência de monitoramento clínico ambulatorial constante são algumas das mudanças impostas por esse tratamento, o qual impacta em todas as instâncias da vida do paciente renal crônico (Jesus et al., 2019).

Por isso a importância do diagnóstico realizado nos estágios iniciais da doença, o qual possibilita a adoção de medidas preventivas que retardam ou interrompem a progressão da DRC. O encaminhamento imediato para o nefrologista e a educação pré-diálise, por exemplo, são condutas essenciais para a diminuição da morbidade e da mortalidade desses pacientes (Bastos \& Kirsztajn, 2011).

Assim, considerando os impactos do diagnóstico tardio e a alta prevalência da DRC, a sua investigação em pacientes que apresentam fatores de risco é imprescindível para o diagnóstico precoce, visto que se trata de uma patologia que cursa de forma assintomática inicialmente.

Considerando o atraso diagnóstico da DRC como determinante de um pior prognóstico, haja vista o prejuízo do tempo de tratamento conservador somado ao início precoce de TRS, seria o diagnóstico precoce um facilitador de um melhor prognóstico para esses pacientes?

Tendo isso em vista, o presente estudo tem como objetivo analisar a quantidade de pacientes com diagnóstico de DRC de etiologia indeterminada, bem como o tempo decorrido entre a data de diagnóstico da DRC e o início da TRS, salientando a importância do rastreio de DRC em pacientes com fatores de risco, conhecidos ou não.

\section{Metodologia}

Trata-se de um estudo observacional, descritivo e retrospectivo, baseado na análise de variáveis quantitativas (Estrela, 2018). Foi realizado no município de Aparecida de Goiânia - Goiás, tomando como fonte de informação os prontuários dos pacientes da Milli Clínica Nefrológica referentes ao período de 2018 a 2021.

O estudo foi realizado no município de Aparecida de Goiânia - Goiás. Esta cidade está localizada na região metropolitana de Goiânia, capital goiana. Segundo o último censo do Instituto Brasileiro de Geografia e Estatística (IBGE) de 2010, a população é de 455.657 pessoas. Compõem a população de estudo todos os pacientes admitidos na diálise da Milli Clínica Nefrológica no período de janeiro de 2018 a março de 2021.

A amostra do estudo foi por conveniência. É um estudo que analisou prontuários de pacientes que foram admitidos nos últimos 3 anos e 2 meses, perfazendo, em média, 59 pacientes por ano, totalizando 177 pacientes.

O processo de coleta de dados foi por meio da análise de prontuários, buscando as características sociodemográficas: sexo, faixa etária e procedência, assim como as variáveis clínicas: fatores de risco prévios para DRC, tempo de tratamento conservador da DRC, data de diagnóstico da DRC, data de admissão à TRS e etiologia da DRC. Foi disponibilizada aos pesquisadores uma sala reservada e restrita na Milli Clínica Nefrológica para que a coleta de dados fosse realizada.

Pacientes que foram admitidos na terapia de substituição renal, de ambos os sexos, maiores de 18 anos, na Milli Clínica Nefrológica em Aparecida de Goiânia - Goiás no período de 2018 a 2021.

Pacientes que receberam alta ou foram à óbito no período de estudo, pois não estavam em processo de diálise no momento da coleta de dados, e pacientes cujos prontuários apresentassem erros graves de preenchimento ou que estivessem 
incompletos.

Os dados foram transcritos para planilha em Programa MS Excel Office XP. Posteriormente, foi feita uma análise estatística descritiva dos dados, sendo estes descritos em frequências relativa e absoluta.

O presente estudo foi aprovado pelo Comitê de Ética em Pesquisa - UniEVANGÉLICA de acordo com o parecer de número 4.461.132, seguindo a Resolução 466/2012 do Conselho Nacional de Saúde (CNS), que dispõe sobre pesquisas com seres humanos.

\section{Resultados}

Foram analisados 177 prontuários no Centro de Diálise da Milli Clínica Nefrológica no período de janeiro de 2018 a março de 2021.

Dos 177 prontuários avaliados inicialmente, como mostrado na figura 1, a maioria era procedente de Aparecida de Goiânia ( $\mathrm{n}=116 / 65,5 \%$ dos pacientes), seguido por Senador Canedo ( $\mathrm{n}=40 / 22,5 \%$ dos pacientes) conforme a Figura 1.

Figura 1. Número de pacientes de acordo com sua procedência em relação ao período de janeiro de 2018 a março de 2021 no Centro de Diálise da Milli Clínica Nefrológica, Aparecida de Goiânia - Goiás.

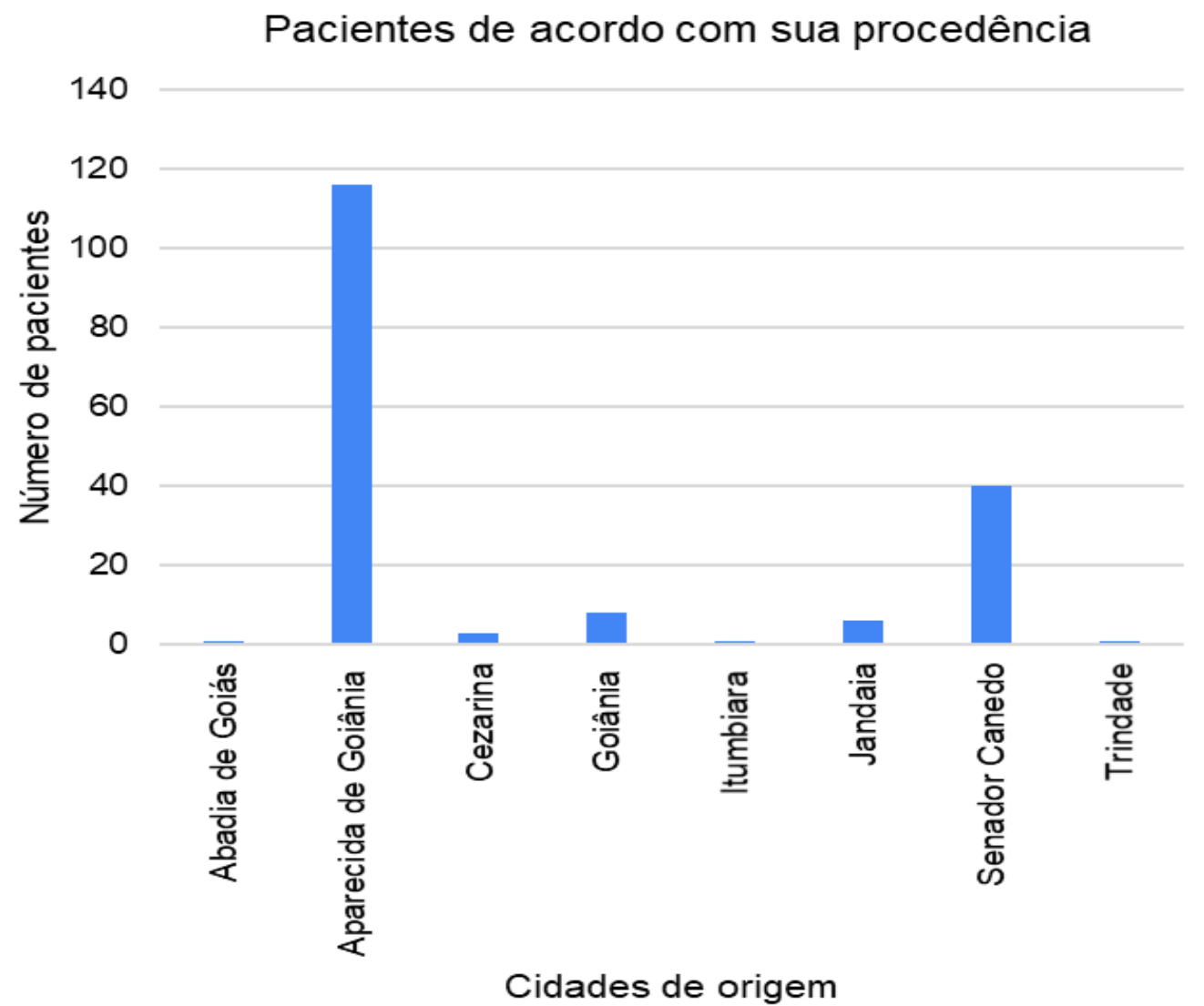

Fonte: Elaborada pelos autores.

Além disso, na Figura 2, é mostrado que boa parte dos pacientes se apresentava na idade de 50-59 anos (n=49/27,6\% dos pacientes), seguido pela faixa etária de 60-69 anos $(n=43 / 24,2 \%$ dos pacientes) e de 40-49 anos ( $n=39 / 22,0 \%$ dos pacientes), enquanto os extremos de idade possuíam uma diminuição de casos de DRC, com as idades de 20-29 anos com n=6 e de 90-99 $\operatorname{anos} \operatorname{com} n=1$. 
Figura 2. Número de pacientes de acordo com sua faixa etária em relação ao período de janeiro de 2018 a março de 2021 no Centro de Diálise da Milli Clínica Nefrológica, Aparecida de Goiânia - Goiás.

\section{Pacientes de acordo com sua faixa etária}

60

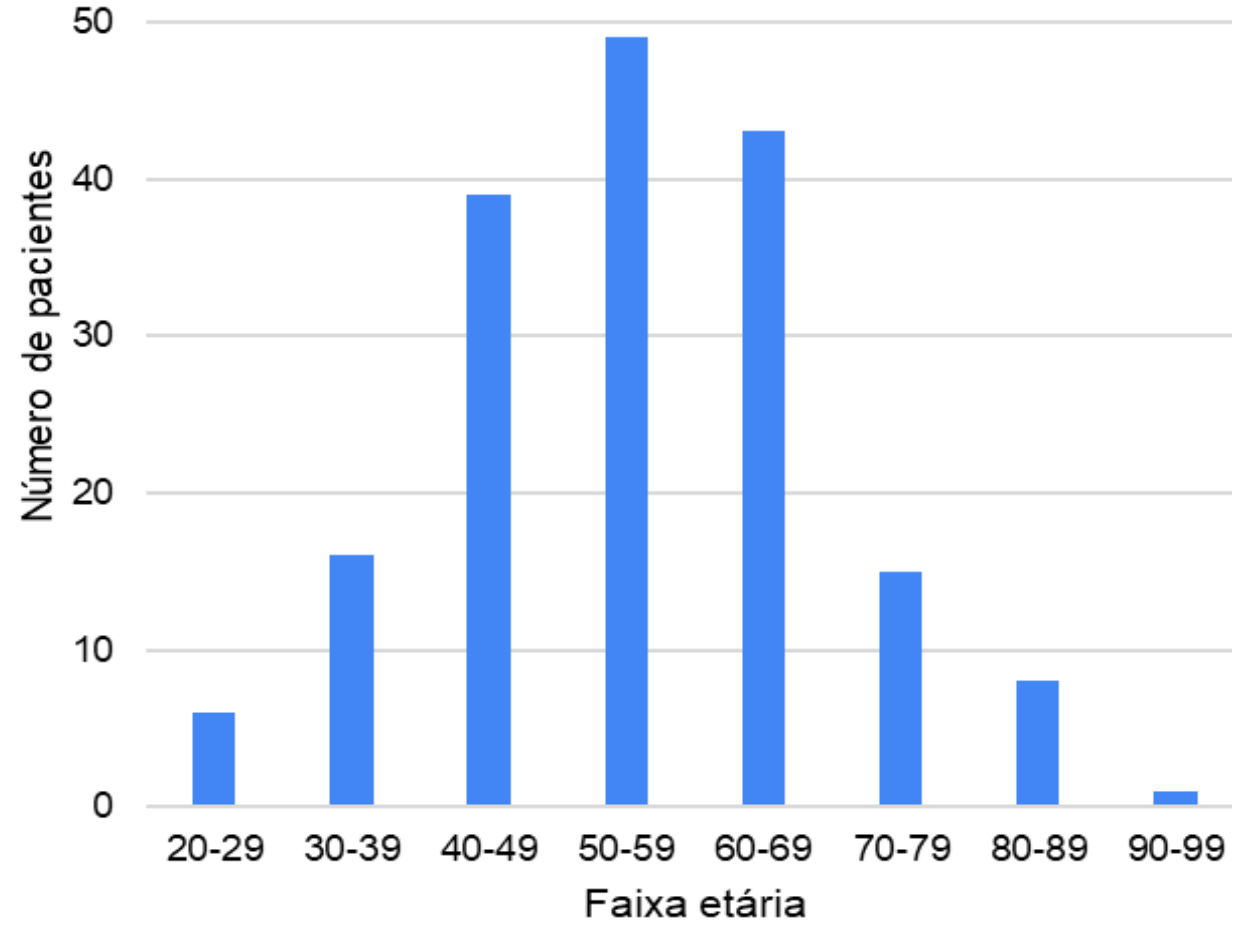

Fonte: Elaborada pelos autores.

Foi observado também que a maioria dos pacientes era do sexo masculino ( $n=105 / 59,3 \%$ dos pacientes), comparado com os pacientes de sexo feminino ( $\mathrm{n}=72 / 40,7 \%$ dos pacientes $)$, como exibido na Figura 3.

Figura 3. Número de pacientes de acordo com o sexo em relação ao período de janeiro de 2018 a março de 2021 no Centro de Diálise da Milli Clínica Nefrológica, Aparecida de Goiânia - Goiás.

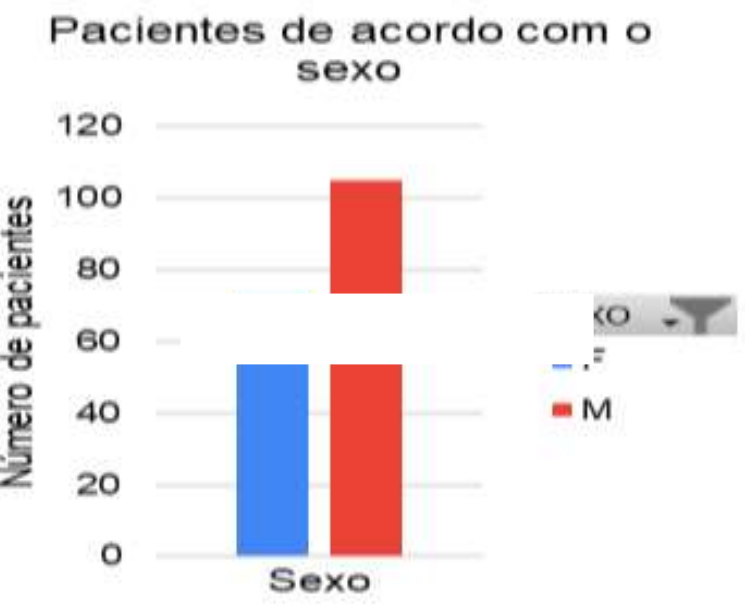

Fonte: Elaborada pelos autores. 
Dentro dos pacientes analisados, foram estabelecidos os fatores de riscos prévios para DRC, dentre eles HAS, diabetes mellitus (DM), litíase, uso de medicamentos/drogas, infecção do trato urinário (ITU) de repetição, idiopática ou indeterminado e por outros fatores (tabagismo, etilismo, rins policísticos, causas cardiovasculares e endócrinas, HIV, lúpus eritematoso sistêmico, gota, bexiga neurogênica, neoplasias, hiperplasia prostática benigna, glomerulopatias, nefrectomia, hepatite C, fimose grave e rim único).

Analisando esses fatores, foi possível estabelecer que a maioria possuía pelo menos um fator de risco ( $\mathrm{n}=158 / 89 \%$ dos pacientes), sendo o mais prevalente a HAS ( $\mathrm{n}=118 / 68 \%$ dos pacientes), definido como o principal fator de risco para o desenvolvimento da DRC. Ademais, 19 pacientes apresentaram fator de risco idiopático, não sendo capaz de definir a etiologia da sua DRC, o que também é algo considerável, já que em 11\% dos pacientes de uma clínica de hemodiálise não foi possível definir o que provocou a DRC (Figura 4).

Figura 4. Número de pacientes de acordo com seus fatores de risco em relação ao período de janeiro de 2018 a março de 2021 no Centro de Diálise da Milli Clínica Nefrológica, Aparecida de Goiânia - Goiás.

\section{Pacientes de acordo com seus fatores de riscos}

140

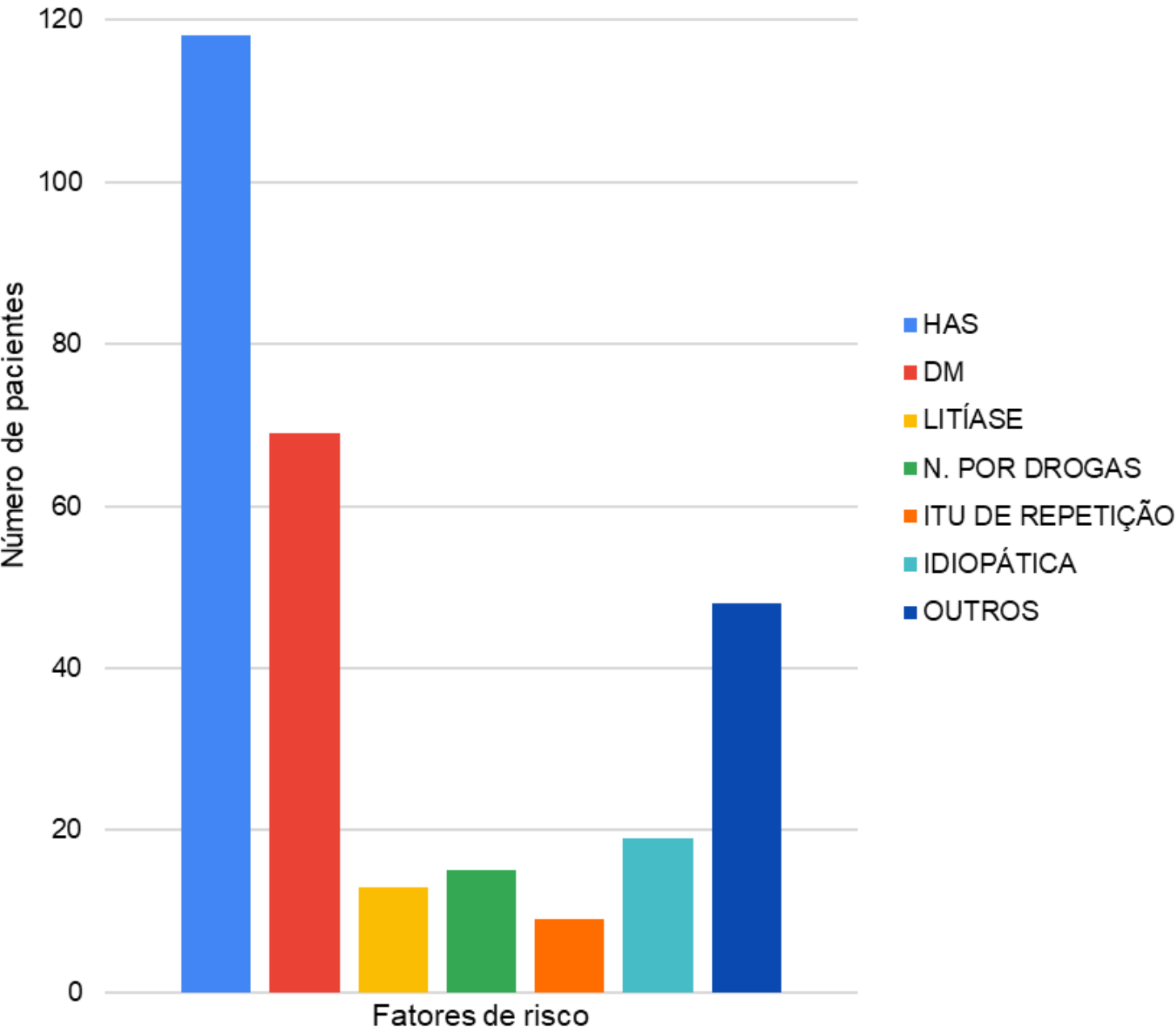

Fonte: Elaborada pelos autores. 
Os resultados mostram que há uma prevalência dos pacientes que não realizaram tratamento conservador ( $\mathrm{n}=112 / 63,2 \%$ dos pacientes), seguido pelo extremo de 22 pacientes (12,4\%) que realizaram o tratamento conservador há 5 anos ou mais, enquanto outros são classificados por menos de um ano até menos de 4 anos, e outros por não terem dados disponíveis em relação ao tempo de tratamento, como exibido na Figura 5. Além disso, muitos pacientes tiveram o diagnóstico de DRC no dia em que foi iniciada a TRS, o que demonstra que vários pacientes não tiveram um diagnóstico precoce para conseguirem realizar o tratamento conservador da DRC como forma de evitar ou retardar a necessidade de implantação de TRS.

Figura 5. Número de pacientes de acordo com o tempo de tratamento conservador em relação ao período de janeiro de 2018 a março de 2021 no Centro de Diálise da Milli Clínica Nefrológica, Aparecida de Goiânia - Goiás.

\section{Pacientes de acordo com o tempo de tratamento conservador}

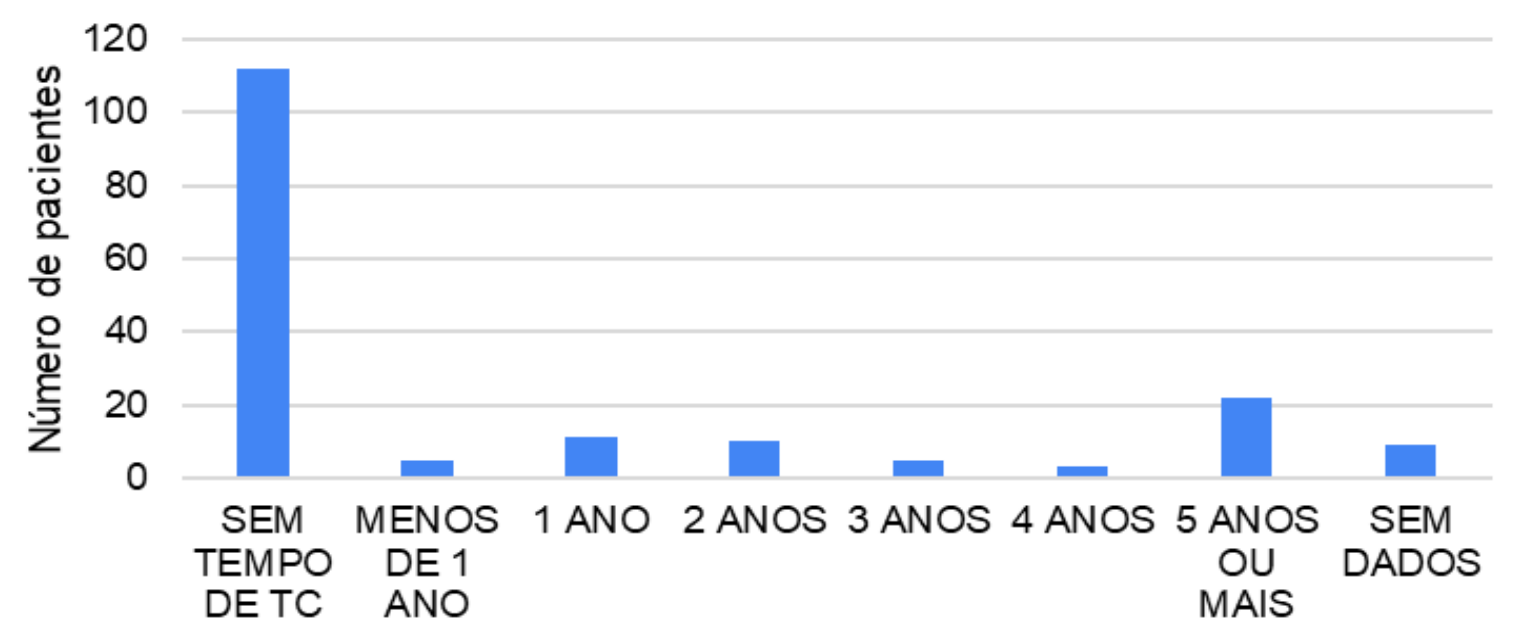

Tempo de tratamento conservador

Fonte: Elaborada pelos autores.

\section{Discussão}

Diante dos resultados obtidos com o presente estudo, percebe-se que a faixa etária prevalente dos pacientes com DRC transitou entre 50 e 59 anos de idade $(n=49)$. Além disso, foi verificado que a maioria dos pacientes acometidos pela doença era do sexo masculino $(n=105)$. Embora o estudo de Tesfaye et al. (2020) esteja condizente em relação ao sexo mais acometido, a faixa etária não está de acordo. O estudo citado revelou que a maioria dos pacientes analisados eram homens, brancos e com idade entre 40 e 49 anos.

Já os resultados observados por Selvarajh et al., (2016) evidencia uma prevalência de DRC entre homens com idade entre 30 e 60 anos, intervalo mais próximo do resultado obtido no presente estudo. Entretanto, a pesquisa realizada por Moura et al. (2015) já mostra que o diagnóstico médico de DRC foi estatisticamente semelhante entre os sexos masculino e feminino, não mostrando diferenças significativas da doença entre os dois sexos em uma amostragem executada por um conglomerado em três estágios, cujos setores censitários foram as unidades primária.

Em relação aos dados levantados acerca dos fatores de risco de DRC, a maioria apresentou pelo menos um fator de risco. A HAS se comportou como o principal fator de risco, sendo identificada em $68 \%$ dos pacientes da amostra. Tal fato converge com o resultado encontrado por Vanelli et al. (2020) no seu estudo sobre a suscetibilidade de DRC em uma amostra representativa, no qual é observado um elevado risco de DRC aliado à alta prevalência de HAS. Neves et al. (2020) também 
afirma que em relação a etiologia da doença renal primária, as principais encontradas são a HAS (34\%) e diabetes (31\%), segundo dados de um levantamento em unidades de diálises do Brasil cadastradas na Sociedade Brasileira de Nefrologia em 2018. Enquanto Marinho et al. (2017) sustenta grande importância que disparidades socioeconômicas, raciais e de gênero são também fatores determinantes para a doença.

Tratando-se de HAS, cabe salientar que essa patologia pode se comportar tanto como causa quanto consequência da DRC. Independentemente da cronologia, a HAS aumenta de forma progressiva e paralela ao declínio da função renal (Barroso et al., 2021).

A HAS-S, definida como aumento da pressão arterial resultante de causa identificável, traz a DRC como um dos principais fatores de risco, o que evidencia a importância do rastreio da DRC nos pacientes hipertensos. Vale lembrar que, segundo o estudo de Barroso et al. (2021), cerca de 90\% dos pacientes com DRC em estágio 5 apresentam HAS. Os mecanismos fisiopatológicos subjacentes ao aparecimento dos sintomas e a interação com a função renal ainda não são totalmente compreendidos. Espera-se que, com a progressão da doença, a manifestação subjetiva dessa condição aumente (Janmaat et al., 2020). Contudo, o interstício tubular contribui para a progressão da DRC em todos os estágios. A ativação e desdiferenciação das células do túbulo proximal mediam múltiplos componentes da resposta fibrogênica. Um fator crítico na progressão da fisiopatologia é a hipertrofia dos néfrons remanescentes. Todos os componentes da fisiopatologia representam funções tubulares normais que saem do controle em uma resposta adaptativa à lesão (Schnaper, 2017).

Ainda sobre os fatores de risco, sobressai-se o fato de 19 pacientes terem apresentado causa indeterminada quanto a etiologia da DRC. Embora não seja um número tão alarmante quanto o encontrado por Ruppel et al. (2018), cujo estudo revelou uma porcentagem de 48,8\% para os casos de DRC de causa indefinida, ainda se trata de um achado que reflete a falha da deteç̧ão precoce da doença, de seus fatores de risco e, consequentemente, do manuseio desses pacientes.

Da amostra, 122 pacientes não realizaram tratamento conservador. Vale lembrar que o tratamento conservador possibilita a adoção de medidas preventivas que retardam ou até mesmo interrompem a progressão da doença (Bastos \& Kirsztajn, 2011).

Além disso, outro fato importante é o número de pacientes diagnosticados com DRC no dia do início da TRS (n=36). Esse achado condiz com as Diretrizes Clínicas para o Cuidado ao paciente com Doença Renal Crônica - DRC no Sistema Único de Saúde/ Ministério da Saúde (2014) que afirmam que uma doença de evolução assintomática, como a DRC, é imprescindível a importância de seu rastreio. Desse modo, a avaliação clínica da função renal é central para a prática da medicina e a TFG é amplamente aceita como o melhor índice de função renal na saúde e na doença (Stevens \& Levey, 2009).

Santos e Montes Neto (2016) apresentam a importância do rastreamento da DRC no estudo "Aplicação do Questionário SCORED para Rastreamento da Doença Renal Crônica entre Pacientes Hipertensos e Diabéticos". Idealizado nos Estados Unidos, o Screening For Occult Renal Disease (SCORED) é um questionário que rastreia e alerta sobre o risco de DRC em fase inicial. Sua vantagem reside no fato de ser composto por questões aptas a serem respondidas por leigos, sem necessidade de exames laboratoriais. No Brasil, validado em amostra de funcionários da Universidade Federal de Juiz de Fora, o SCORED demostrou sensibilidade de $80 \%$ e valor preditivo negativo de $97 \%$ para detecção da DRC.

Em concordância com o presente estudo, a análise feita por Santos e Montes Neto (2016) traz a importância de se rastrear a DRC por se tratar de uma doença que ainda é diagnosticada tardiamente, principalmente pelo fato de as pessoas com fatores de risco não terem a função renal avaliada. Esse cenário impede que medidas preventivas sejam implementadas. 


\section{Conclusão}

Avaliar a função renal de pacientes com fatores de risco, em especial a HAS, é imprescindível para retardar uma possível TRS. Afinal, foi constatado que a HAS age tanto como causa quanto como consequência da DRC, além de possuir um papel indiscutível em sua fisiopatologia.

A partir da coleta de dados e do estudo dos referenciais teóricos, nota-se que a DRC ainda é uma doença subdiagnosticada. A falta de investigação da etiologia dos sintomas iniciais impede a detecção precoce da doença e a instituição de um tratamento conservador, afetando a vida do paciente nos âmbitos físicos, psicológicos, sociais e financeiros. Frisa-se, portanto, a necessidade de a classe médica atentar-se a possibilidade de uma DRC ao se deparar com um paciente com fatores de risco para esta doença.

Finalizamos este trabalho com a intenção de incentivar e favorecer novos caminhos e pesquisas, diante de uma realidade em que a Medicina é uma ciência em constante evolução, e buscar sempre por conhecimento significa prezar pela vida de nossos pacientes da melhor forma. Os resultados dessa pesquisa permitirão uma abordagem cada vez mais multidisciplinar da doença renal crônica, buscando a atenção plena as suas múltiplas etiologias.

\section{Agradecimentos}

Agradecemos a Milli Clínica Nefrológica pela permissão e oportunidade de realizarmos nosso estudo em seu centro de diálise. Agradecemos, sobretudo, a nossa professora-orientadora, Dra. Rhaisa Macedo, que nos guiou desde o princípio na elaboração da pesquisa e mostrou-se exemplo de profissional ética e humana.

\section{Referências}

Amaral, T. L. M., Amaral C. A., de Vasconcelos, M. T. L., \& Monteiro, G. T. R. (2019) Prevalência e fatores associados à doença renal crônica em idosos. Revista de Saúde Pública, 53(44), 1-11. doi:10.11606/S1518-8787.2019053000727

Barroso, W. K. S., Rodrigues C. I. S., Bortolotto, L. A., Mota-Gomes, M. A., Brandão, A. A., de Magalhães, A. D., Machado, C. A. et al. Diretrizes Brasileiras de Hipertensão Arterial - 2020. Arq. Bras. Cardiol., 116 (3), 516-558. doi: 10.36660/abc.20201238

Bastos, M. G., \& Kirsztajn, G. M. (2011). Doença renal crônica: importância do diagnóstico precoce, encaminhamento imediato e abordagem interdisciplinar estruturada para melhora do desfecho em pacientes ainda não submetidos à diálise. Brazilian Journal of Nephrology, 33(1), 93-108. doi:10.1590/S010128002011000100013

Bikbov, B., Purcell, C., Levey, A. S., Smith, M., Abdoli, A., Abebe, M., Adebayo, O. M. et al. (2020) Global, regional, and national burden of chronic kidney disease, 1990-2017: a systematic analysis for the Global Burden of Disease Study 2017. The Lancet, 395 (10225), 709-733. doi:10.1016/S0140-6736(20)300453

Brasil. Ministério da Saúde. Secretaria de Atenção à Saúde. Departamento de Atenção Especializada e Temática (2014) Diretrizes Clínicas para o Cuidado ao paciente com Doença Renal Crônica - DRC no Sistema Unico de Saúdel Ministério da Saúde. Secretaria de Atenção à Saúde. Departamento de Atenção Especializada e Temática. Brasília: Ministério da Saúde.

Estrela, C. (2018). Metodologia Científica: Ciência, Ensino, Pesquisa. Editora Artes Médicas.

Janmaat, C. J., van Diepen, M., Meuleman, Y., Chesnaye, N. C., Drechsler, C., Torino, C. et al. (2021). Kidney function and symptom development over time in elderly patients with advanced chronic kidney disease: results of the EQUAL cohort study. Nephrology Dialysis Transplantation, 36(5), 862-870. doi: $10.1093 / \mathrm{ndt} / \mathrm{gfz} 277$

Jesus, M. N., de Souza, G. F., Rodrigues, C. M., Almeida Neto, O. P., Rodrigues, D. D. M., \& Cunha, C. M. (2019) Quality of life of individuals with chronic kidney disease on dialysis. Brazilian Journal of Nephrology, 41(3), 364-374. doi:10.1590/2175-8239-JBN-2018-0152

Kirsztajn, G. M., Salgado Filho, N., Draibe, S. A., de Pádua Netto, M. V., Thomé, F. S., Souza, E., \& Bastos, M. G. (2014) Leitura rápida do KDIGO 2012: Diretrizes para avaliação e manuseio da doença renal crônica na prática clínica. Brazilian Journal of Nephrology, 36(1), 63-73. doi:10.5935/01012800.20140012

Marinho A. W. G. B., Penha, A. P., Silva, M. T., \& Galvão, T. F. (2017) Prevalência de doença renal crônica em adultos no Brasil: revisão sistemática da literatura. Cadernos Saúde Coletiva, 25(3), 379-388. doi:10.1590/1414-462X201700030134

Moura, L. D., Andrade, S. S. C. D. A., Malta, D. C., Pereira, C. A., \& Passos, J. E. F. (2015). Prevalência de autorrelato de diagnóstico médico de doença renal crônica no Brasil: Pesquisa Nacional de Saúde, 2013. Revista Brasileira de Epidemiologia, 18, 181-191. doi:10.1590/1980-5497201500060016 
Research, Society and Development, v. 10, n. 14, e523101422278, 2021

(CC BY 4.0) | ISSN 2525-3409 | DOI: http://dx.doi.org/10.33448/rsd-v10i14.22278

Neves, P. D. M. D. M., Sesso, R. D. C. C., Thomé, F. S., Lugon, J. R., \& Nasicmento, M. M. (2020). Censo Brasileiro de Diálise: análise de dados da década 2009-2018. Brazilian Journal of Nephrology, 42, 191-200. doi: 10.1590/2175-8239-JBN-2019-0234

Oliveira, C. M., dos Santos, E. B. A., Bermude, B. E. B. V., Ferreira, L. F., Tizzot, E. A., \& Mourão Júnior, C. A. (2019) A importância do médico de atenção primária no rastreamento e diagnóstico precoce da doença renal crônica. Revista Ciências em Saúde, 9(2), 3-8. doi: 10.21876/rcshci.v9i2.728

Ruppel, P., Felipe, C. R., Pestana, J. O. M., Hiramoto, L. L., Viana, L., Ferreira, A., \& Aguiar, W. et al. (2018) A influência de fatores clínicos, ambientais e socioeconômicos na sobrevida de cinco anos após o transplante renal. Jornal Brasileiro de Nefrologia, 40 (2), 155-161. doi:10.1590/2175-8239-JBN-3865

Santos, P. R., \& Montes Neto, V. L. (2016). Aplicação do questionário SCORED para rastreamento da doença renal crônica entre pacientes hipertensos e diabéticos. Cad. Saúde Colet., 24 (4), 428-434. doi:10.1590/1414-462X201600040178

Schnaper, H. W. (2017). The tubulointerstitial pathophysiology of progressive kidney disease. Advances in chronic kidney disease, 24(2), 107-116. doi: 10.1053/j.ackd.2016.11.011

Selvarajah, M., Weerantunga, P., Sivayoganthan, S., Rathnatunga, N., \& Rajapakse, S. (2016) Clinicopathological correlates of chronic kidney disease of unknown etiology in Sri Lanka. Indian Journal of Nephrology, 26(5), 357-363. doi:10.4103/0971-4065.167280

Stevens, L. A. \& Levey, A. S. (2009). Measured GFR as a confirmatory test for estimated GFR. Journal of the American society of nephrology, 20(11), 23052313. doi: 10.1681/ASN.2009020171

Tesfaye, W. H., McKercher, C., Peterson, G. M., Castelino, R. L., Jose, M., Zaidi, S. T., \& Wimmer, B. C. (2020) Medication Adherence, Burden and HealthRelated Quality of Life in Adults with Pre dialysis Chronic Kidney Disease: A Prospective Cohort Study. International Journal of Environmental Research and Public Health, 371 (17), 1-13. doi:10.3390/ijerph17010371

Vanelli, C. P., de Paula, R. B., Costa, M. B., Bastos, M. G., Miranda, L. S. P. \& Colugnati, F. A. B. (2017) Doença renal crônica: suscetibilidade em amostra representativa de base populacional. Revista de Saúde Pública, 52(68), 1-4. doi:10.11606/S1518-8787.2018052017410

Vieira, A. C., Malagutti, W., Rodrigues,.F. S. M., de Deus, R. B., Barnabe, A. S., Francisco, L., \& Ferraz, R. R. N. (2010). Avaliação dos principais fatores etiológicos em indivíduos portadores de insuficiência renal crônica em hemodiálise. ConScientiae Saúde, 9(3), 462-468. Disponível em: https://www.redalyc.org/articulo.oa?id=92915180017 